\section{Rapidly Rotating Nuclei}

\section{J.D. Garrett, G.B. Hagemann, and B. Herskind, Roskilde}

(The Niels Bohr Institute, University of Copenhagen)

High-spin physics has attracted considerable attention in recent years. Heavy ion beams allow nuclei to be formed with the maximum angular momentum that such systems can sustain, adding a new dimension to the study of nuclear dynamics.

\section{Formation and Decay}

Beams of $5 \mathrm{MeV} / \mathrm{u}$ particles of mass 30-50 are now available in many laboratories and can, through the fusion reac- tion produce rapidly rotating nuclei. Cross-sections for the process approach one barn. An example is the argon on tin reaction ${ }^{124} \mathrm{Sn}\left({ }^{40} \mathrm{Ar}, \mathrm{xn}\right)$ ${ }^{164-x} \mathrm{Er}$ shown in Fig. 1. The upper portion of this figure illustrates how the colliding nuclei fuse and form a composite system. Products with angular momenta $\leq 60 \mathrm{~h}$ have a large probability of surviving fission and forming a compound nucleus with rotational frequencies, $\hbar \omega$, up to $\approx 0.75 \mathrm{MeV}$ (i.e. $\approx 2 \times$ $10^{20}$ rotations per second). Such highly-

Fig. 1 - Schematic representation depicting the formation and decay of the compound nucleus. The insert shows a statistical model calculation of the decay process. There is a small probability for $\gamma$-ray emission in competition with neutron evaporation due to the enhanced Giant Dipole Resonance strength (GDR).



FORMATION

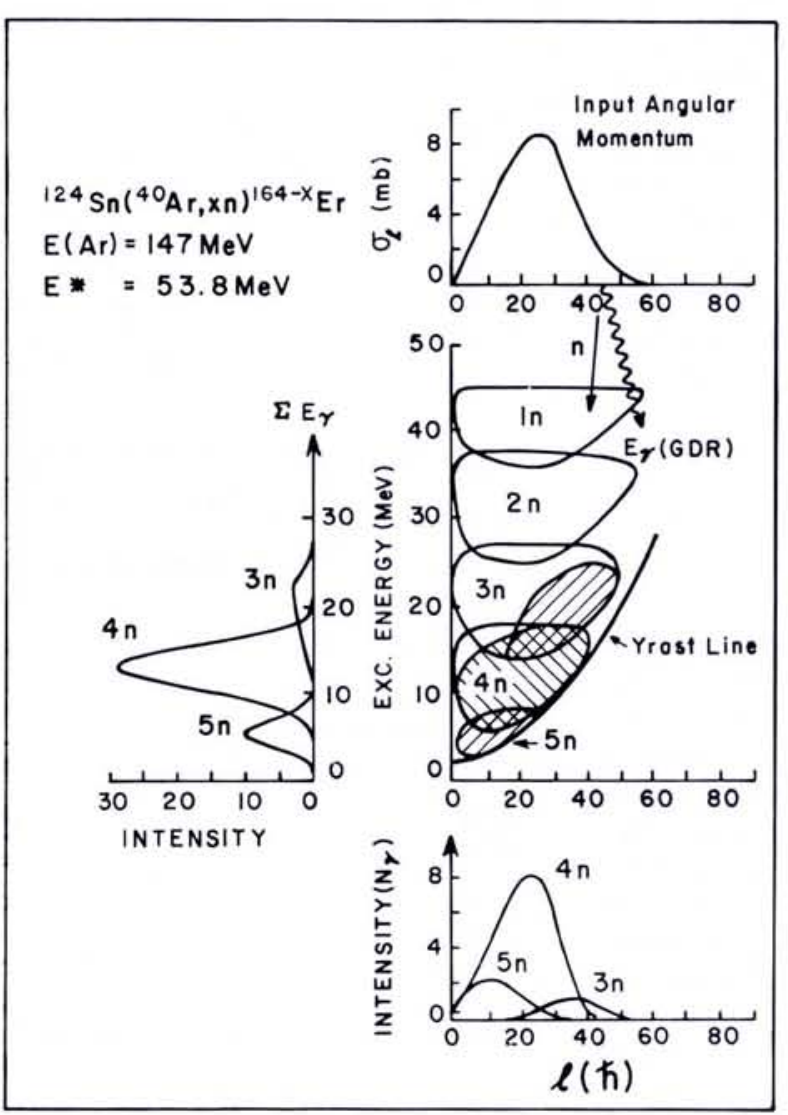

excited nuclei evaporate neutrons over a characteristic time of $10^{-19} \mathrm{~s}$. Neutron emission is efficient in reducing the excitation energy of the system, but it removes only a small fraction of the angular momentum. When the nucleus has "cooled" to an excitation energy within one neutron binding energy $(\approx 8$ $\mathrm{MeV}$ ) of the so-called yrast line, it will continue to "cool" by emitting "statistical" dipole and collective quadrupole gamma radiation on a characteristic time scale of $10^{-15} \mathrm{~s}$. The yrast (or dizziest) line delimits the maximum angular momentum for a given excitation energy. As the excitation energy approaches this line, quadrupole gamma radiation which is more efficient in reducing angular momentum, dominates the decay process which is completed in about $10^{-9} \mathrm{~s}$ during which the nucleus has rotated about $10^{11}$ times, i.e. nearly as many times as the Earth since its creation.

A statistical calculation of the process is shown in the insert of Fig. 1 where the starting point of the gamma-ray cascade, after the emission of 3,4 or 5 neutrons is indicated by the shaded regions. These "entry regions" also are projected on the energy and angular momentum axes, illustrating how such experimental information can be obtained by measuring the total cascade energy in a " $4 \pi$ detector" and the number of gamma rays per cascade, $M$. For rare earth nuclei which predominantly emit quadrupole radiation, the angular momentum, $\ell$, is related to $M$ by $\ell=2(M-3)$.

Large multi-detector systems (crystal balls) have been constructed recently at the Oak Ridge National Laboratory and the Max-Planck-Institut in Heidelberg for such measurements, although most of the data presented in this review was obtained using a multi-detector system that became operational at Daresbury Laboratory in January 1983 , or by a predecessor which was located at the Niels Bohr Institute in 1981-82. The present version consists of a mini crystal ball of about 50 bismuth germanate (BGO) detectors, which subtend a solid angle approaching $4 \pi$. Six Ge detectors, Compton suppressed by means of large $\mathrm{Nal}$ shields, are situated outside the ball looking through small gaps between the BGO elements. By using the 15 possible pairings of the suppressed $\mathrm{Ge}$ detectors, the energy-correlation of gamma-ray pairs can be measured with high efficiency, low background and high resolution. The total cascade energy and the gamma-ray multiplicity are measured simultaneously in the ball for each $\mathrm{Ge}$ Ge event. 

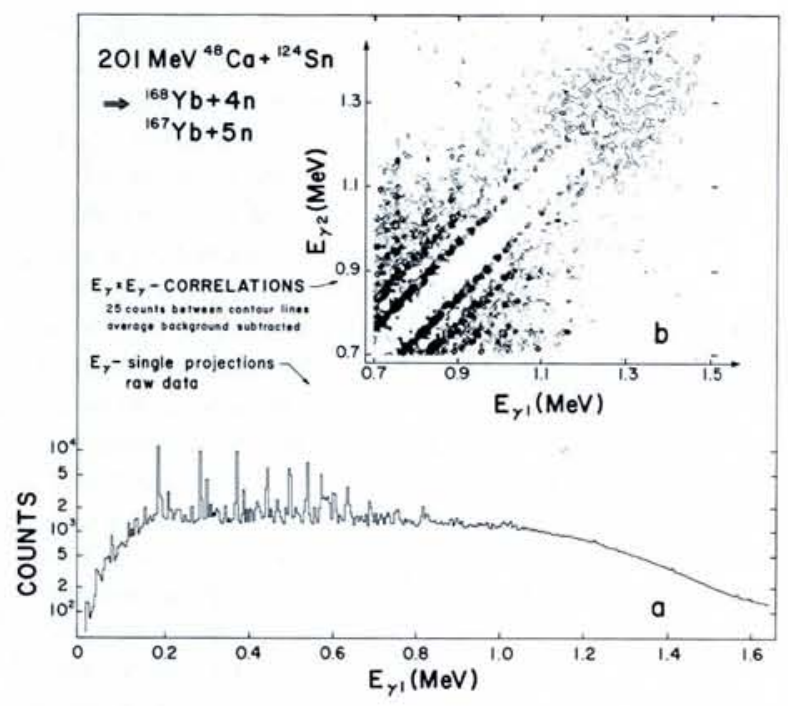

\section{Gamma-ray Spectra}

A Compton-suppressed $\mathrm{Ge}$ spectrum for the ${ }^{48} \mathrm{Ca}+{ }^{124} \mathrm{Sn}$ reaction is shown in Fig. $2 a$ on a logarithmic scale. Three types of gamma-ray transitions can be distinguished:

i) lower energy discrete gamma-ray lines corresponding to transitions along strongly populated near-yrast rotational sequences based on low-lying intrinsic configurations;

ii) unresolved collective quadrupole transitions that dominate the spectrum below $1.4 \mathrm{MeV}$;

iii) a "tail" of high energy statistical dipole transitions which mainly remove energy from the nucleus.

Decay schemes up to $I \approx 40$ have been constructed from discrete line coincidence data. Such a scheme for a single decay sequence in ${ }^{168} \mathrm{Hf}$ is shown in Fig. 3a. Nuclear structure information for the highest angular momenta, however, can be obtained by studying the correlations between the energies of two coincident gamma rays, Fig. 2 b. Distinct features are isolated in such an $E_{\gamma 1}-E_{\gamma 2}$ correlation spectrum whereas the corresponding uncorrelated spectrum is nearly featureless, Fig. $2 a$. In the present case a distinct valley flanked by several parallel ridges is observed along the $E_{\gamma 1}=E_{\gamma 2}$ diagonal. Such structure is typical of decay via a multitude of rotational bands based on different intrinsic nuclear configurations.

\section{Independent Particle Motion in a Rota- ting Deformed System}

The nuclear potential describes the many-body system as the interaction of independent particles with an average field, $h_{\text {def }}$, of shape reflecting the nuclear deformation. A pairing potential, $h_{\text {pair }}$, is added to this field as an effective two-body interaction, scattering pairs of particles between the eigenstates of the
Fig. 2 - a) The spectrum of $\gamma$-rays observed in a Compton-suppressed Ge detector after the reaction ${ }^{124} \mathrm{Sn}\left({ }^{48} \mathrm{Ca}, \mathrm{xn}\right){ }^{172-x} \mathrm{Yb}$ at $201 \mathrm{MeV}$ bombarding energy.

b) The $E_{\gamma 1} \times E_{\gamma 2}$ correlation spectrum for the same reaction in the region $0.7<E_{\gamma}<1.5 \mathrm{MeV}$. been subtracted from the measured excitation energy. Calculations of the energies of the completely paired ground state and the lowest two-quasiparticle excitation of $i_{13 / 2}$ neutrons are also shown in Fig. 3b. The $i_{13 / 2}$ neutrons with $\ell_{, j}=6,13 / 2$ have the largest particle angular momentum in rare earth nuclei; as a result these are the particles most strongly influenced by the rotation-induced forces. For no rotation, the completely paired ground state is lowered relative to the two-quasineutron states by twice the pairing gap energy. Under rotation, the energy of the highly alignable (i.e. large $j_{x}$ ) two-quasineutron configuration is reduced, as a result of the

Fig. 3 - Experimental energies of the ground state decay sequence of ${ }^{168} \mathrm{Hf}$ shown (a) in the laboratory frame versus spin $l$, and (b) in the rotating intrinsic frame versus rotational frequency, $h \omega$. The insert in part (a) shows a conventional decay scheme. The solid line in part (b) is a cranking model calculation based on systematic parameters. In the intrinsic frame the $e^{\prime}$ at $h \omega=O$ of the aligned 2-quasineutron configuration is approximately equal to twice the pairing gap energy $\triangle$. The experimental aligned angular momentum $i=-d e^{\prime} / d(h \omega) \approx$ $9 \mathrm{~h}$ for this configuration. mentary excitations become independently moving quasiparticles which are mixtures of particles and holes. Such pair correlations yield a "superfluid" nuclear state with a resulting reduced moment of inertia.

The intrinsic rotational energy is expressed by $\omega j_{x}$, where $j_{x}$ is the component of the particle angular momentum in the direction of the rotational axis. This third field which at the highest rotational frequencies can be of a similar magnitude to the nuclear shell effects, quench the pairing interaction. Consequently, important information on the microscopic basis of pairing correlations is obtainable from spectroscopic studies of rapidly rotating nuclei.

In the remainder of this short review we shall illustrate how such information can be obtained from spectra such as that shown in Fig. 2.

\section{Configuration Dependent Pairing}

Experimental energies corresponding to the ground state decay sequence of ${ }^{168} \mathrm{Hf}$, converted to excitation energies in the rotating intrinsic frame are shown in Fig. $3 b$ as a function of the angular frequency of rotation, $h \omega$. As the independent particle hamiltonian given by eq. (1) is valid in the intrinsic frame, we have chosen to express the experimental data in that frame in order to preserve the independent particle picture. The energy associated with rotation has therefore
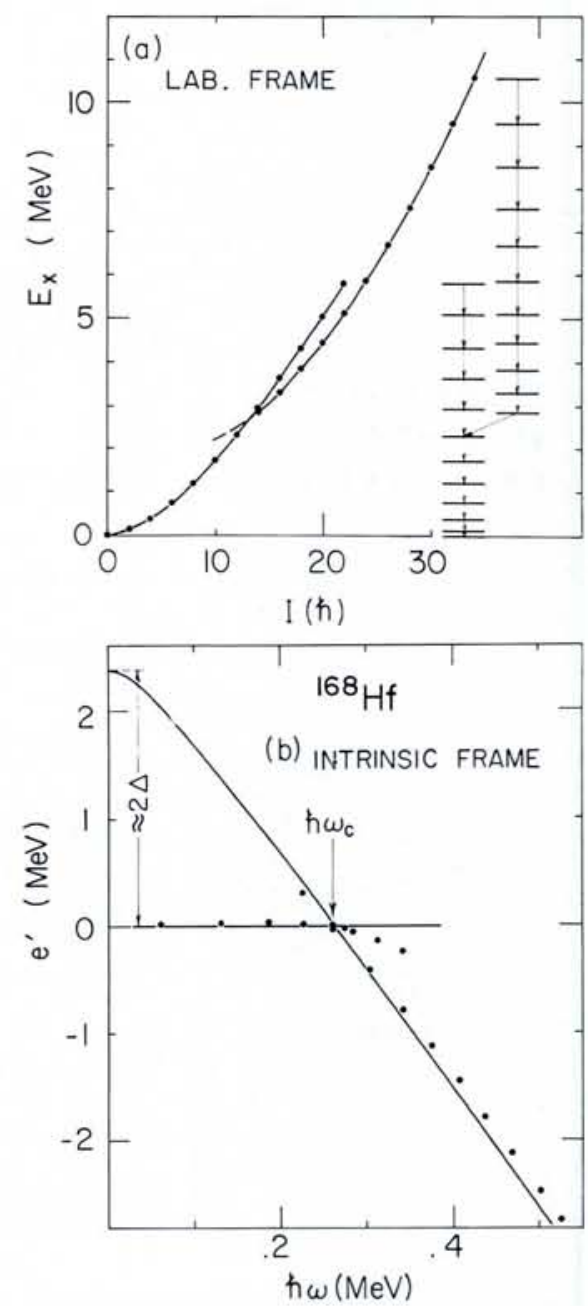


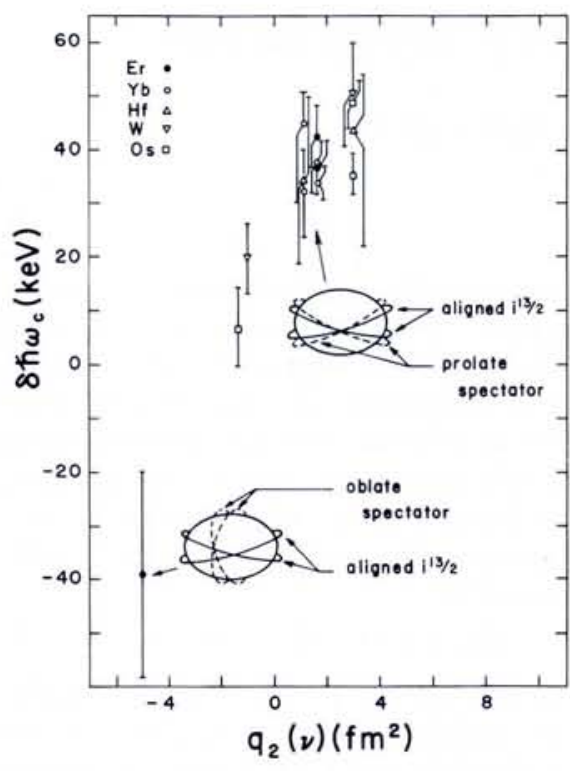

Fig. 4 - Correlation between the shift in band crossing frequencies, $\delta \hbar \omega_{c} \equiv$ $\hbar \omega_{c}($ even- $N)-\hbar \omega_{c}($ odd $-N)$, and the $q_{2}(v)$ $\equiv\left\langle v / r^{2} Y_{20} / v\right\rangle$, of the orbit of the spectator quasineutron. The overlap of a prolate aligning $i_{13 / 2}$ quasiparticle with a prolate and an oblate spectator, respectively, is sketched in connection with the appropriate data points.

rotation-induced forces. At $h \omega=0.26$ $\mathrm{MeV}$, the energy associated with these forces on the $i_{13 / 2}$ two-quasineutron configurations is equal in magnitude, but opposite in sign to the pairing gap energy. Thus the energy of this twoquasineutron state becomes degenerate with the paired ground-state configuration. Since the gamma-ray cascade tends to follow the configuration which is lowest in energy, at this band crossing or "backbending" the cascade will change from a rotational band based on the aligned two-quasineutron configuration to a band based on the paired ground state configuration. This is equivalent to the change from the normal to the superfluid state in rotating liquid ${ }^{4} \mathrm{He}$ or in a superconductor subject to an applied magnetic field.

Such band crossing frequencies, $\hbar \omega_{c^{\prime}}$ based on the alignment of a pair of $i_{13 / 2}$ quasineutrons can be established from the experimental data, see Fig. 3b, so it is possible to determine the magnitude of the pairing gap energy, on a relative scale. Band crossings corresponding to the alignment of this pair of $i_{13 / 2}$ quasineutrons are observed systematically at smaller frequencies in most decay sequences of nuclei with an odd number of neutrons. This decrease in $h \omega_{c}$ indicates a reduced pairing associated with the aligned pair of $i_{13 / 2}$ quasineutrons when a "spectator" quasineutron is present. Thus pairing contributions are partially "blocked" in the odd- $N$ nucleus. How- ever, when the "blocked" orbital is oblate, i.e. is strongly aligned with the nuclear symmetry axis perpendicular to the rotational axis (Fig. 4), the reduction of $h \omega_{c}$ is smaller. The observed configuration dependence of the shift in $h \omega$ between neighbouring even- $N$ and odd- $N$ isotopes is illustrated in Fig. 4 indicating that the pairing contributions to the aligned $i_{13 / 2}$ neutron orbitals are state dependent. A large overlap between the "blocked" valence orbital and the aligned configuration produces a large contribution to pairing and a small overlap produces a small contribution. From such data it is possible to differentiate between the microscopic pairing contributions of various configurations. The nucleus with its three-dimensional shape imposed by a "finite" number of nucleons is a unique quantum system for the study of such shape-dependent pairing effects.

The larger band crossing frequency for the most oblate configuration (Fig. $4)$, indicates a negative pairing contribution when this configuration is "blocked". This is, of course, unphysical indicating the presence of other effects.
Realistic calculations in which the influence of the "blocked" configuration on the nuclear shape is considered, are in quantitative agreement with the data. The band crossing systematics illustrate the type of spectroscopic information which is obtainable from such high spin data. Data are also available as a function frequency. A more general analysis in terms of residual interactions between the constituent quasiparticles is needed for a proper interpretation, but is beyond the scope of this introduction.

Moments of Inertia at the Highest Spins Above spin forty there is insufficient intensity in any specific rotational band to observe the discrete transitions, even with the best Compton-suppressed detectors. Detailed features, however, can be isolated from $E_{\gamma 1}-E_{\gamma 2}$ correlation spectra such as that shown in Fig. 2. In these spectra the width of the central valley,

$$
W=E_{\gamma}(I+2)-E_{\gamma}(I-2),
$$

is an average measure of the moment of inertia of the nucleus rotating at $h \omega=$ $E_{y} / 2$.



The Daresbury Laboratory, an establishment of the Science and Engineering Research Council, situated in the Cheshire countryside, has Research Associateship positions in Nuclear Theory.

The Laboratory provides central facilities for Universities in the UK: a 20 MV Tandem Van de Graaff Nuclear Structure Facility and a dedicated Synchrotron Radiation Source of $x$-ray and ultra-violet radiation used for physics, chemistry and biology experiments.

The work in the Nuclear Theory Group consists of studies of high spin states in nuclei and the investigation of the reaction mechanisms in the collisions of heavy ions and nuclei. The successful applicants will be expected to take a strong interest in the experiments currently performed and those which are planned on the Nuclear Structure Facility. Preference will be given to candidates with experience in the structure of nuclei, or reaction aspects of heavy ion collisions.

Applicants (male or female) should possess a $\mathrm{PhD}$ degree, or expect to obtain one during 1984 in theoretical nuclear physics.

Appointments will be made at salaries between $£ 6,985$ and $£ 10,686$ per annum depending on age, ability and experience. The posts will be available for a fixed term of three years and are superannuable.

CLOSING DATE: 31st March 1984

For further information write to or telephone

Dr. M. A. Nagarajan (0925) 65000 Extension 211

Applications should be sent, together with curriculum vitae and the addresses of two referees, quoting reference DL/ 837 to:

The Personnel Officer, Daresbury Laboratory, Science \& Engineering Research Council, Daresbury, Warrington, Cheshire WA4 4AD. England. 
The energy of a nucleus in an intrinsic configuration, $v$ with associated energy $E_{v}$ and moment of inertia $J$ is

$$
\begin{aligned}
E & =\left(h^{2} / 2 J\right) R^{2}+E_{\mathrm{v}} \\
& =\left(h^{2} / 2 J\right)(I-i)^{2}+E_{\mathrm{v}}
\end{aligned}
$$

Here the total angular momentum $I$ is assumed to be composed of two components (Fig. 1): that generated by collective rotations about an axis perpendicular to the nuclear symmetry axis, labelled $\mathbf{R}$, and the angular momentum of the excited nucleons, $\mathbf{j}$. The total component of $\mathbf{j}$ aligned with the rotational axis $\mathrm{x}$ is designated by $i=\Sigma j_{\mathrm{x}}$. The first derivative of the rotational energy with respect to spin defines a kinematic moment of inertia, $J^{(1)}$ :

$$
d E / d l=\left(h^{2} / J^{(1)}\right)(I-i)
$$

A moment of inertia, $J^{(2)}$ can also be defined in terms of the second derivative:

$$
d^{2} E / d l^{2}=\hbar^{2} / J^{(2)}
$$

This dynamical moment of inertia is related to the difference in transition energies between neighbouring transitions in a rotational sequence. Therefore, it is the $J^{(2)}$ that is measured by the width of the central valley in the $E_{\gamma 1}-E_{\gamma 2}$ correlation plots.

The width of the valley in Fig. 2 is nearly constant over a large frequency range, $0.35<\hbar \omega<0.6 \mathrm{MeV}$, indicating a nearly constant moment of inertia. The observed value is only slightly smaller than the moment of inertia of a rigid prolate ellipsoid with deformation $\beta=0.3$. Similarly the narrow ridges imply that the spread in the values of $J^{(2)}$ for the various configurations rotating at these frequencies is small. Such large nearly constant values of the moment of inertia for ${ }^{167},{ }^{168} \mathrm{Yb}$ suggest that at large frequencies where the pairing correlations are expected to be small, these nuclei behave much like macroscopic rotors.

\section{Perspectives}

As discrete gamma-ray spectroscopy approaches the regime where pairing correlations appear to become less important, it is interesting to speculate on the future of such studies. Much of the recent discrete line studies have addressed the question of the frequency and configuration dependence of pairing correlations. In the region where such correlations are minimal, more specific information on other spectroscopic quantities, e.g. nuclear shapes and the details of the nuclear potential, is already emerging.

\section{REFERENCE}

Additional reference to the spectroscopy of rapidly rotating nuclei can be found in: Nuclear Physics A400 (1983) 113c.

\title{
Supersymmetry in Nuclei
}

\author{
F. lachello, New Haven, CT \\ (Yale University)
}

Symmetry considerations are playing an increasingly important role in all fields of physics. In particular, in the last few years, the concept of dynamic symmetry has emerged as a powerful tool in the study of complex systems. This concept has been further extended to include a new, more elaborate type of symmetry, called supersymmetry. Ap plications of dynamic symmetry considerations to nuclear spectra have provided (i) a deeper understanding of properties of nuclei, (ii) an elegant and very concise classification scheme of these properties and finally (iii) led to the discovery of experimental examples of dynamic supersymmetries in physics.

\section{Dynamic Symmetries}

The property that makes a dynamic symmetry a powerful tool in analyzing complex systems is that, when such a symmetry exists, the energy levels (and other properties) can be written in closed form. These closed forms, containing only the quantum numbers labelling the states, can be easily checked by experiment. In addition to providing concise and elegant classification schemes, dynamic symmetries give clues to the underlying dynamics and almost invariably lead to a fundamental understanding of the problem.

The oldest and most familiar example of a dynamic symmetry is provided by the hydrogen atom. Pauli (1926) and Fock (1935) showed that one could write a closed form for the energy levels

$$
E\left(n, \ell, m_{\ell}\right)=-A / n^{2} \text {, }
$$

because of the existence of a dynamic symmetry, O(4). However, dynamic symmetries did not receive much atten- tion until the early 60 s, when they were applied to the study of the low-lying spectra of hadrons. As is well known, it was suggested at that time by GellMann and Ne'eman that these spectra could be classified as representations of an SU(3) group. This led to an energy formula (the Gell-Mann-Okubo mass formula)

$$
E\left(I, I_{3}, Y\right)=a+b Y+c\left[I(I+1)-\frac{Y^{2}}{4}\right]
$$

which has $S U(2) U(1)$ dynamic symme try, and later to an understanding of the underlying dynamics in terms of quarks.

\section{Dynamic Symmetries in Nuclei}

In recent years, the concept of dynamic symmetries has been applied to the study of complex nuclei, and has led to major advances in this field. The applications are based on a nuclear model, called the interacting boson model, that I now briefly review. In 1974 , Arima and I') suggested that the lowlying states of nuclei with an even number of protons and neutrons could be described by assuming that these nuclei are constructed out of boson-like building blocks. These building blocks were later interpreted as being highly correlated pairs of protons and neutrons $^{2}$ similar to the Cooper pairs of the electron gas. Of all the possible values of the total angular momentum, $J$, of the pairs, only $J=0$ and $J=2$ were retained. The $J=0$ pairs were called s-bosons, while the $J=2$ pairs were called $d$ bosons, as shown in Fig. 1. Since the single component of the s-boson and the five components of the d-boson span a six-dimensional space, we suggested that the low-lying spectra of even-even nuclei be classified as

Fig. 1 - Schematic representation of the structure of even-even (left) and even-odd (right) nuclei. In the low-lying states of even-even nuclei all protons and neutrons are paired together; in odd-even nuclei complete pairing is impossible and in addition to $s$ - and $d$ bosons there is an unpaired nucleon (fermion).

(a) even-even nuclei

protons

d-bosons
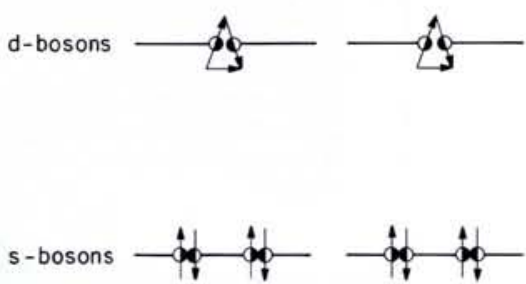

(b) even-odd nuclei

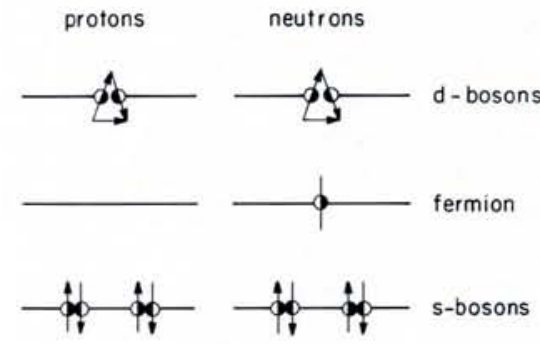

